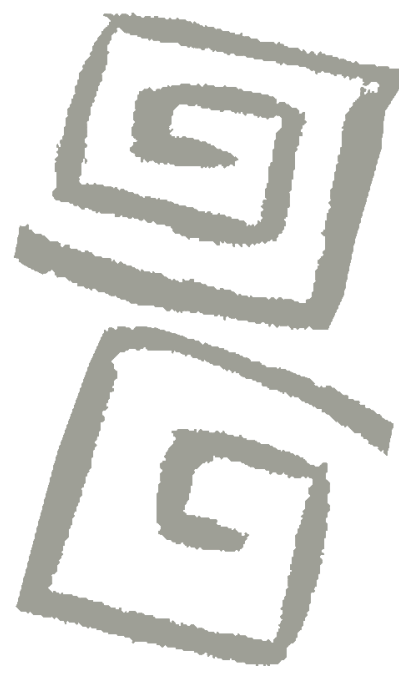

\title{
El desafío de la inclusión en salud mental: análisis de un centro comunitario y su trabajo sobre los vínculos sociales
}

\author{
The challenge of inclusion in mental health: an \\ analysis of a community center and its work with \\ social bonds
}

Sara Ardila-Gómez ${ }^{1}$, María Isabel Hartfiel², Marina A. Fernández³ , Guadalupe Ares Lavalle ${ }^{4}$, Mariana Borelli ${ }^{5}$, Alicia Stolkiner ${ }^{6}$

${ }^{1}$ Psicóloga. Doctora en Salud Mental Comunitaria. Becaria Posdoctoral, Consejo Nacional de Investigaciones Científicas Técnicas (CONICET), sede Departamento de Salud Comunitaria, Universidad Nacional de Lanús, Buenos Aires, Argentina. $₫$ (iD

2Licenciada en Sociología. Coordinadora, Centro Comunitario Libremente, Hospital Interzonal José A. Estéves, Buenos Aires, Argentina. $\square$ (iD)

${ }^{3}$ Licenciada en Psicología. Becaria Doctoral,

Universidad de Buenos Aires, Ciudad Autónoma de Buenos Aires, Argentina. $\square$ (iD)

${ }^{4}$ Licenciada en Psicología. Docente Investigadora, Departamento de Salud Comunitaria, Universidad Nacional de Lanús, Buenos Aires, Argentina. $\triangle$ (iD

5Licenciada en Psicología. Psicóloga, Programa de Rehabilitación y Externación Asistida, Hospital Interzonal José A. Estéves, Buenos Aires, Argentina. $\square$ (iD)

${ }^{6}$ Licenciada en Psicología. Diplomada en Salud

Pública. Profesora Titular, Departamento de Salud Comunitaria, Universidad Nacional de Lanús. Profesora Titular, Facultad de

Psicología, Universidad de

Buenos Aires, Argentina. $\square$ i
RESUMEN La transformación de la atención en salud mental supone tomar como eje de trabajo la inclusión social, considerando tanto los beneficios que la vida comunitaria trae para las personas con trastorno mental, como los que podría tener para las demás personas de la comunidad. Con el fin de analizar los alcances de la inclusión en salud mental, se entrevistó a 45 asistentes a un centro comunitario vinculado a un programa de externación de un hospital psiquiátrico, el cual busca explícitamente propiciar la inclusión social. Se indagó sobre las posibles transformaciones en las relaciones sociales entre personas externadas y otros miembros de la población, a partir de la interacción cotidiana y sostenida en dicho centro. Los resultados sugieren que uno de los beneficios que encuentran los asistentes es el establecimiento de vínculos, ligados al apoyo informal. A su vez, se observan transformaciones positivas en las ideas respecto a las personas con "enfermedad mental", aunque las mismas parecieran estar más del lado de la integración que de la inclusión social.

PALABRAS CLAVES Integración a la Comunidad; Salud Mental; Promoción de la Salud; Argentina.

ABSTRACT Social inclusion is a key component of transformations in mental health care, because it takes into account the benefits of community life for both those with mental illness and the other members of the community. In order to understand the scope of inclusion within mental health, 45 participants of a community center linked to a psychiatric hospital discharge program which explicitly seeks to provide social inclusion were interviewed. The possible changes in social relationships between users and other community members based in their sustained daily interactions in the community center were explored. Results suggest that the building of social bonds, as part of informal support networks, is one of the benefits of attending the community center. Positive changes in ideas regarding people with "mental illness" were also observed, although these ideas seemed to be more connected to the notion of integration than to social inclusion.

KEY WORDS Community Integration; Mental Health; Health Promotion; Argentina. 


\section{INTRODUCCIÓN}

Es ampliamente reconocido que las relaciones sociales son uno de los principales aspectos de la vida de las personas que se ven afectados por la presencia de un trastorno mental severo(1)[a]. Sin embargo, las instituciones diseñadas para el tratamiento de tales afecciones -los hospitales psiquiátricos- contribuyeron durante mucho tiempo al empobrecimiento relacional de las personas atendidas, en tanto generaban distanciamiento de los lugares de origen y aislaban a las personas internadas de espacios cotidianos de socialización, lo que producía efectos iatrogénicos. A partir de ello, los movimientos de reforma en salud mental han procurado modificar dicha situación, tomando como uno de sus ejes de acción el mejoramiento de los vínculos de las personas con trastorno mental severo mediante su recuperación, ampliación y fortalecimiento ${ }^{(2)}$.

Ahora bien, los movimientos de transformación de la atención en salud mental desarrollados en los últimos 70 años alrededor del mundo dieron lugar a una gran heterogeneidad de servicios, como hospitales de día y de noche, centros de día, clubes de día, centros comunitarios, centros de salud mental comunitaria, por mencionar solo algunos de la amplia gama de instituciones existentes. Durante ese lapso de tiempo, la conceptualización sobre tales servicios y, en particular, sobre sus objetivos se fue modificando y se comenzó a concebir que el fin de estas instituciones estaba más del lado de la recuperación de derechos ciudadanos que de la rehabilitación ${ }^{(3)}$. Este cambio conceptual implicaba que los servicios debían modificar su foco -el cual tradicionalmente se había puesto en las personas con trastorno mental severo- para centrarse en las relaciones sociales entre tales personas y los demás integrantes de las comunidades de las cuales eran parte. El trabajo desde un enfoque relacional supone reconocer a todos los actores significativos del proceso en estudio y analizar las interacciones que se dan entre ellos $^{(4)}$.
De este modo, el foco en lo relacional llevó a comprender que la inclusión de personas con trastorno mental severo en la vida cotidiana no solo las beneficiaba -en la medida en que les posibilitaba fortalecer sus redes sociales- sino que, a su vez, enriquecía a los demás integrantes de la comunidad, en tanto les permitía ampliar sus redes sociales y les daba la oportunidad de vivenciar y practicar la convivencia con la diferencia como parte fundamental de la vida en sociedad. Sin embargo, pese a ser este un objetivo desde los servicios, la investigación académica no ha acompañado suficientemente dichas transformaciones, y los estudios realizados, cuando se han orientado hacia los integrantes de las comunidades receptoras, han tendido a centrarse en su aceptación de las personas con trastorno mental severo $y$, en mucho menor medida, en los efectos o transformaciones en ellas mismas o en su red social ${ }^{(5,6)}$.

Cabe señalar que este cambio de foco en la intervención es parte del debate que se ha producido entre las nociones de integración e inclusión social en diversos ámbitos, como el de la discapacidad, la educación, y la salud mental, debate que se ha tornado confuso en la medida en que ambos términos suelen utilizarse como sinónimos, pese a corresponder a paradigmas diferentes. La integración social supone que una persona a la cual se la haya identificado con alguna diferencia respecto a un grupo mayoritario -por ejemplo, con una discapacidad- pueda ser en algún aspecto miembro activo de ese conjunto social, aunque las estructuras de interacción entre los participantes se mantengan sin grandes modificaciones. En sentido estricto, la integración propone la apertura de espacios de socialización a los "diferentes", dado que consiste en:

Participation by a (devalued) person or persons in social interactions and relationships with non-devalued citizens that are culturally normative both in quantity and quality, and that take place in normative activities and in valued, or at least normative, settings or context. ${ }^{(7 p .18)}$ 
[La participación de una o varias personas (devaluada) en interacciones sociales y relaciones con ciudadanos no-devaluados que son culturalmente normativas en cantidad y calidad, y que tienen lugar en actividades, escenarios o contextos valorados].

Por su parte, la inclusión social considera que la discapacidad surge de las limitaciones e inequidades que produce la sociedad al estar diseñada de un modo homogéneo, basado en una idea de "normalidad", por lo que las intervenciones no apuntan a la adaptación de los diversos, sino a la modificación de los ambientes, en los que todas las personas son una parte definitoria para que sea posible la participación y la igualdad de oportunidades de los miembros de la sociedad ${ }^{(8)}$.

En este sentido, la Convención Internacional sobre los Derechos de las Personas con Discapacidad ${ }^{(9)}$ pone en discusión el concepto de integración social, asociándolo al modelo rehabilitador -el cual entendía a las personas con discapacidad como sujetos a ser rehabilitados para alcanzar un ideal- y propone, en cambio, el concepto de inclusión, fundamentado en el modelo social, el cual considera que la discapacidad se produce porque la sociedad no tiene la forma de alojar a una persona con características diferentes y por ello no la habilita a desarrollar sus capacidades potenciales. Así, la discapacidad se produce entre la persona y la sociedad. Desde esta perspectiva, las personas con discapacidad pueden aportar a la sociedad en igual medida que el resto de los individuos, enfatizando la valoración de cada sujeto y el respeto de las diferencias. Sus principios son la vida independiente, la no discriminación y la accesibilidad universal, desde un enfoque de derechos humanos ${ }^{(10)}$. De este modo, los problemas de la inclusión social son aquellos que afectan severamente la calidad de vida de una parte de la población, a nivel material y simbólico, derivados de las desventajas que surgen por estar excluidos de las oportunidades compartidas por otros ${ }^{(11)}$.

En el campo de la salud mental, el cambio de orientación hacia la inclusión social ha encontrado como obstáculo las dificultades de los servicios y, dentro de estos, de los trabajadores del campo, para pensar en términos de categorías relacionales. Incluso, dentro de los servicios denominados comunitarios, una característica frecuente es centrarse en los "pacientes", y enfocar su mirada en uno de los componentes de un campo de intervención que por definición debería ser relacional.

Dentro del terreno de la producción de conocimientos, a la ya escasa tarea de sistematización y evaluación de lo que se produce en salud mental, algo que también sucede es que los indicadores y las formas de pensar instituidas tienden a concentrarse en lo individual, es decir, en "el paciente". Ello resulta particularmente problemático para los servicios de carácter comunitario, los cuales son los que tienen más dificultades para dar cuenta de su quehacer, dado que sus acciones tienden a incluir, de diversas maneras, a otros actores más allá de los "pacientes". A nivel regional, cabe destacar los intentos de sistematización de experiencias comunitarias coordinados por la Organización Panamericana de la Salud ${ }^{(12)}$, así como los desarrollos en Brasil y Chile, países en los que el despliegue de servicios comunitarios ha sido parte de un proceso de reforma de carácter nacional| ${ }^{(13,14)}$. En el caso de Argentina, esta falta de información genera una vulnerabilidad importante para este tipo de servicios, aspecto que es crucial en el escenario actual del país dado que, en el marco de la implementación de la Ley Nacional de Salud Mental|(15), se vive una puja entre diversos actores del campo, en la cual el escaso conocimiento sistemático sobre los servicios comunitarios se torna un elemento en la disputa de poder. De allí que, en los últimos años, diversos actores han incrementado el interés por dar cuenta de lo que se hace en este tipo de servicios ${ }^{(16)}$.

A partir de lo anterior, desde hace ocho años se viene desarrollando una línea de investigación acerca de la evaluación de servicios comunitarios de salud mental. En este trabajo se presentan los resultados de un estudio realizado entre 2011 y 2014 en el marco de esta línea de investigación, titulado "Evaluando las transformaciones producidas 
por la externación de pacientes psiquiátricos en las comunidades en las cuales viven: percepciones desde la comunidad", en el cual se indagó sobre las transformaciones en las actitudes de la comunidad en la cual pasaban a vivir personas que habían estado internadas de forma prolongada en un hospital psiquiátrico. El estudio tuvo dos etapas: en la primera, se centró en los vecinos de las casas de convivencia en las cuales vivían personas usuarias de un programa de externación ${ }^{(17,18)}$ $y$, en la segunda, se analizó en profundidad el centro comunitario de dicho programa, al cual asisten tanto personas externadas como así también otros miembros de la comunidad, bajo el supuesto de que el centro comunitario es un lugar privilegiado de interacción, es decir, de transformaciones para todas las personas que allí concurren. Aquí se presentan los resultados de esta segunda etapa, cuyo objetivo fue describir y analizar estas posibles transformaciones en las relaciones sociales entre personas que padecieron prolongadas internaciones en instituciones psiquiátricas monovalentes y otros miembros de la población, a partir de la interacción cotidiana y sostenida en un centro comunitario que tiene como una de sus misiones principales promover la inclusión social en su comunidad.

\section{Características del centro comunitario}

El centro comunitario en el cual se realizó el estudio funciona desde hace 15 años y es parte del Programa de Rehabilitación y Externación Asistida del Hospital Interzonal José A. Estéves, un hospital psiquiátrico monovalente de mujeres ubicado en la zona sur del Gran Buenos Aires. El programa tiene dos componentes: uno de acompañamiento a la externación enfocado en las usuarias, y otro de promoción de la salud orientado a toda la comunidad. Los objetivos iniciales de este centro fueron, por un lado, constituirse en espacio de interacción entre las usuarias del programa y la comunidad a partir del desarrollo de actividades culturales y de capacitación, funcionando así como dispositivo de inclusión social y, por otro lado, ser la sede física del programa por fuera del hospital psiquiátrico.

Las actividades ofertadas por el centro se dirigen a toda la comunidad, incluyendo a las usuarias del programa, lo cual significa que el objeto de acción e intervención del centro no son solo las usuarias externadas, sino toda la comunidad. Dicho en términos de los fundadores del programa: "no se trata de pedirle a la comunidad que sea solidaria con las usuarias y las acepte, sino de ofrecerle a la comunidad actividades que sean de su interés, desde una perspectiva de promoción de la salud". Es importante contextualizar el nacimiento del centro comunitario en la época inmediatamente anterior a la crisis social y económica que vivió la Argentina en el año 2001; crisis que, junto al sufrimiento que vivía la población, trajo aparejada la revitalización de los espacios de vinculación, intercambio y solidaridad entre las personas.

Así, en el centro comunitario funcionan en paralelo actividades propias del componente de acompañamiento a la externación, dirigidas exclusivamente a las usuarias: atención individual y grupal, asambleas de usuarias y reuniones de equipo; con actividades dirigidas a toda la comunidad: talleres, cursos de capacitación y actividades culturales. De este modo, el uso del espacio en el centro es compartido: en un mismo salón en donde se realizan asambleas con las usuarias, se puede realizar a continuación un taller de tejido abierto a la comunidad. Esto produce que allí necesariamente se encuentren y se crucen las personas de la comunidad con las usuarias del programa, participen o no de manera conjunta en un mismo taller. Compartir el espacio constituye una característica distintiva de este dispositivo frente a otros programas comunitarios de salud mental en el país y es, a su vez, la razón por la cual se lo denomina centro "comunitario", y no "de día" o "de salud mental".

Las actividades del centro se difunden constantemente por distintos medios a fin de incentivar la participación de la comunidad. También se trabaja para fomentar y sobre todo sostener la participación de personas 
con mayor vulnerabilidad (por ejemplo, personas vinculadas a centros de prevención de adicciones, centros de contención y hogares transitorios), debido a que suelen tener mayores dificultades para incluirse en espacios de socialización. Tales personas habitualmente son derivadas al centro por profesionales o por recomendación de otros asistentes. Finalmente, en lo que respecta a la participación de las usuarias del programa, se trabaja junto con los equipos de acompañamiento a la externación, estimulando su participación en las distintas actividades de acuerdo a sus intereses y, desde el año 2013, se indica a las usuarias recién externadas la obligatoriedad de asistir a algún taller durante los primeros dos meses de su vida fuera del hospital, luego de lo cual es decisión de ellas seguir concurriendo o no. Finalmente, cabe señalar que algunas usuarias del programa son a su vez trabajadoras del centro, en tareas administrativas, de limpieza o asistentes de los profesores de los talleres.

Las actividades ofertadas incluyen taIleres artísticos, de trabajo corporal y deportivo, cursos de capacitación, empresas socioproductivas, actividades sociales y culturales. En los talleres, el trabajo se enfoca en dos objetivos: uno referido al aprendizaje de la actividad concreta del taller (por ejemplo, pintura) y otro dirigido al fomento de la interacción y vinculación entre los participantes. En este sentido, es necesario aclarar que los talleres no son concebidos como espacios terapéuticos, aunque puedan tener efectos de este tipo y, a su vez, que los profesores son personas expertas en el tema específico de cada taller.

\section{METODOLOGÍA}

El estudio tuvo un enfoque mixto, en tanto se produjeron y analizaron datos cuantitativos y cualitativos a fin de lograr un mayor entendimiento del fenómeno investigado ${ }^{(19)}$. Al momento de realizar el estudio, en el centro comunitario funcionaban 18 talleres. Para la selección de los talleres se aplicaron los siguientes criterios de inclusión: aquellos talleres cuyo grupo de asistentes fuera estable, que estuvieran dirigidos a adultos, y que fueran talleres propios de la institución. De este modo, la muestra quedó compuesta por 9 talleres, que contaban con 153 asistentes. Durante la primera semana de agosto de 2013, se realizaron entrevistas estructuradas para lo cual se convocó a 45 personas asistentes al centro y todas aceptaron participar de la investigación.

Las entrevistas se realizaban al inicio de cada taller, de forma individual, a las primeras cinco personas que llegaran y que hubiesen concurrido a ese taller durante más de un mes. La duración de las entrevistas fue en promedio de 20 minutos y el registro se realizó a través de notas tomadas por el entrevistador, que luego fueron transcritas.

Dado que las entrevistas fueron realizadas por integrantes del equipo de investigación que no trabajaban en el centro comunitario, no tenían conocimiento acerca de si la persona entrevistada era o no usuaria del programa de externación. Se informó a cada entrevistado sobre los objetivos del estudio y se le solicitó el consentimiento informado. Con el fin de preservar la confidencialidad, las entrevistas fueron numeradas de acuerdo al orden en el cual fueron hechas.

La entrevista constaba de 27 preguntas, tanto abiertas como cerradas, agrupadas en cuatro secciones: datos sociodemográficos, relación de los entrevistados con el centro, percepciones frente a distintas problemáticas, y percepciones y relaciones con personas con "enfermedad mental". Se utilizó el término "enfermedad mental" ya que, en la prueba piloto del cuestionario, implementada en la primera etapa del estudio, se encontró que era el de mayor comprensión para las personas no vinculadas al campo de la salud mental. Las preguntas fueron categorizadas de manera independiente por al menos dos integrantes del equipo de investigación, discutiéndose posteriormente los casos en los cuales no había acuerdo en la categorización, a fin de definirla. 


\section{RESULTADOS}

Se presentan los resultados de tres de las cuatro secciones de la entrevista, y se excluye la sección de "percepciones frente a distintas problemáticas", puesto que tenía como propósito comparar las respuestas con las obtenidas en el trabajo realizado con los vecinos, aspecto que no se desarrolla en este trabajo.

\section{Datos sociodemográficos}

Un $60 \%$ de las personas entrevistadas asistía al centro hacía más de un año. Un 73\% eran mujeres. Las edades de los entrevistados comprendían un rango de 18 a 77 años, con solo un $9 \%$ de personas mayores de 65 años, es decir, en edad jubilatoria. El nivel de escolaridad fue heterogéneo: un 56\% tenía estudios secundarios completos o superiores y solo un $7 \%$ estudios primarios incompletos o sin escolaridad. En relación con el estado civil, un $20 \%$ estaba casado, un $55 \%$ eran solteros, un $16 \%$ divorciados y un $9 \%$ viudos. La composición de los hogares en los que vivían las personas entrevistadas mostró que un $7 \%$ lo hacía con su familia nuclear, un $73 \%$ con su familia ampliada, un $13 \%$ en hogares unipersonales y un $7 \%$ vivía con otros no familiares.

Respecto de la ocupación, un 33\% de las personas entrevistadas se identificaron como trabajadores en ejercicio, de los que aproximadamente la mitad estaba en relación de dependencia. Las ocupaciones de las restantes fueron: ama de casa $(18 \%)$, estudiantes $(13 \%)$, desempleados $(16 \%)$, pensionados $(11 \%)$ y jubilados $(9 \%)$. Por último, la localidad en la que residían las personas entrevistadas mostró que un $64 \%$ correspondía al mismo municipio en el cual está ubicado el centro, y que el resto se desplazaba desde ocho localidades distintas para acudir a los talleres.

\section{Los vínculos en el centro comunitario}

Un grupo de preguntas de la entrevista indagaba acerca de la relación de las personas con el centro, es decir, cómo habían llegado a este, por qué y para qué iban, de qué les servía asistir y, específicamente, en qué medida ir allí había contribuido al establecimiento de vínculos.

Respecto a las formas en las cuales habían Ilegado al centro, una tercera parte lo hizo por recomendación de un profesional, mientras que otras vías de acceso fueron la recomendación de algún conocido, o vivir cerca. En lo referido a la razón por la cual asistían, la mayoría dijo hacerlo por la actividad central del taller, por ejemplo, aprender a dibujar o aprender un oficio como peluquería. Otro motivo fue la búsqueda de algo que los ayudara frente a ciertos malestares, ligados en su mayoría a la soledad y el aislamiento:

\section{La jubilación parece sensacional, después sentís el vacío. Fui averiguando pero no sabía por dónde empezar. Elegí estos tres talleres y no estoy arrepentida del tiempo que ocupo. (61 años, mujer, taller 4) \\ En principio, para estar con gente. (35 años, mujer, taller 9)}

Una tercera razón para asistir al centro fue la derivación profesional o como forma de tratamiento:

Porque el psiquiatra me lo recomendó como terapia. (61 años, mujer, taller 2)

Necesito un poco, los médicos me recomendaron ir a yoga. (77 años, mujer, taller 4)

Por otro lado, el $84 \%$ de las personas entrevistadas señaló haber realizado vínculos nuevos gracias a su participación en el centro comunitario. De ellas, casi la mitad se relacionaba con esos nuevos vínculos por fuera de las actividades del centro.

Respecto a qué es lo que les gustaba del centro, la mayoría de las respuestas se agruparon en tres categorías: los vínculos, las actividades en sí, y la "integración" social. La categoría de vínculos fue la que agrupó 
la mayor cantidad de respuestas, haciendo mención a la "gente", e incluyendo allí a los compañeros, profesores y demás personal del centro. Sobre la "integración" social, las respuestas agrupadas bajo dicha categoría hicieron referencia explícita a la presencia de "pacientes" o "personas con enfermedad mental", por ejemplo:

El respeto que hay. Están muy bien organizados y la función que cumplen ayudando a las personas con enfermedad mental. (47 años, varón, taller 6)

...que se integra naturalmente, nadie trata distinto por si está mejor o peor. (61 años, mujer, taller 4)

Todo es aprendizaje. Antes los veías aplastados, ahora los ves que vienen. Me entristece un poco. (61 años, mujer, taller 4)

Respecto a lo que motivaba a las personas para seguir asistiendo, la mayoría de las respuestas se agruparon en tres categorías: los vínculos, el aprendizaje de la actividad concreta del taller, y el sentirse bien. Dentro de las respuestas se señaló que ir al centro ayudaba a que las personas salgan de su casa y se encuentren con otros, el compañerismo, el trato en el centro, así como el vínculo con los profesores. Por ejemplo, algunas de las personas entrevistadas señalaron:

Acá encontré gente con la que puedo hablar, tengo amigos. (62 años, mujer, taller 1)

Me da alegría, me hace salir del encierro.

(67 años, mujer, taller 4)

A su vez se indagó sobre aquello que, de acuerdo a las personas entrevistadas, les había servido de asistir al centro. La mayoría de las respuestas se agruparon en dos categorías: una referida al "bienestar" y otra a la "socialización", aunque muchas personas hicieron referencia de manera simultánea a las dos. En relación al "bienestar", los entrevistados lo describieron de maneras diversas, haciendo referencia a cosas tales como:

Para tener la cabeza ocupada en otra cosa, tenía muchos problemas. (42 años, mujer, taller 8)

Estaba deprimida porque perdí un trabajo de 16 años pero me di cuenta que puedo hacer otra cosa. (42 años, mujer, taller 8)

...te sentís bien vos. (67 años, mujer, taller 2)

Para despejarse. (35 años, mujer, taller 3)

Para ver de distintas maneras las cosas.

(34 años, mujer, taller 3)

En cuanto a la categoría de "socialización", las respuestas señalaban que asistir al centro les había servido para conocer gente y establecer vínculos y, a su vez, para mejorar el modo de relacionarse con las personas, haciendo referencia en algunos casos a personas con problemas mentales. Por ejemplo:

Estar con todo tipo de clase de gente. Ayudás y te ayudan. Hay variedad de personas. (54 años, mujer, taller 3)

Me ha servido para sociabilizar. Tengo una amiga. (42 años, mujer, taller 1)

Estar comunicada un poco con la gente, si no me sentía muy sola. (61 años, mujer, taller 2)

\section{Percepciones y relaciones con personas con "enfermedad mental"}

Un $80 \%$ de las personas entrevistadas respondió que conocía a alguien que estuvo internado en un hospital psiquiátrico, más de la mitad de las cuales conoció a esa persona en el centro comunitario. A estos últimos, se les preguntó cómo había sido participar con 
esa persona en las actividades del centro. Cabe resaltar que ninguna respuesta tuvo connotaciones negativas. En algunos casos se refirieron a tales personas en términos de igualdad, encontrándose respuestas como:

...igual que con todas. (19 años, varón, taller 6)

...normal como cualquier otra persona. (31 años, mujer, taller 7)

En otros entrevistados apareció la idea de haberse enriquecido a partir del vínculo:

Me sirvió para aprender, al principio juzgaba, al conocer su historia aprendí lo que pasó. (20 años, varón, taller 6)

Muy lindo, enseña muchas cosas de la vida. (45 años, mujer, taller 7)

En otros casos, hubo reconocimiento de la diferencia, ligada a la idea de tolerancia:

Bien, no te das cuenta, se adaptan al grupo. (47 años, mujer, taller 6)

Yo no me vinculo mucho, ella se vincula conmigo. (61 años, mujer, taller 4)

Bien porque uno como que los acepta. (54 años, mujer, taller 3)

La mayoría de quienes conocieron por primera vez en el centro a una persona que estuvo internada señalaron que su idea sobre tales personas cambió a partir de la interacción, encontrándose en todos los casos respuestas positivas. Es de resaltar que muchas de las personas entrevistadas dijeron que antes tenían una idea de menor aceptación, y que ello se había modificado:

Te hace más humano, ayudás más al conocer más. (54 años, mujer, taller 3)

Vos los tenés más en cuenta. Con nuestro apoyo ellos se pueden integrar en la sociedad. (47 años, mujer, taller 6)
Yo no pensé que me podía relacionar, y sí podés. (47 años, varón, taller 6)

Me ha permitido cambiar las formas de relacionarme de manera más natural. (31 años, mujer, taller 7)

Se preguntó también por lo que pensaban en la actualidad sobre las personas con enfermedad mental $y$, a su vez, por lo que pensaban sobre ellas antes de asistir al centro. Aproximadamente la mitad indicó cambios en su forma de pensar, siendo estos cambios, en su gran mayoría, positivos y solo en un caso, negativo. Sobre los cambios positivos se encontró que el conocimiento de tales personas les hizo aprender cosas:

No las conocía realmente, ahora las quiero y creo que puedo ayudarlas. (42 años, mujer, taller 8)

Antes no pensaba en el tema. Me di cuenta que es importante escucharlas. (35 años, mujer, taller 9)

También, que gracias a conocerlas, se modificaron algunos prejuicios:

Me daban miedo, ahora no porque más o menos conozco. No es nada de lo que imaginaba. (19 años, mujer, taller 8)

...ahora no las juzgo ni me cambio de vereda, cada uno tiene sus dolores. (61 años, mujer, taller 4)

Cabe resaltar que seis de los entrevistados hablaron de la enfermedad mental en primera persona $y$, en todos los casos, hicieron referencia al sufrimiento y a la soledad que les trae la enfermedad, así como al rechazo que perciben por parte de otras personas:

Somos seres que tenemos que estar en constante lucha con nuestra enfermedad. Sufro mucho y tengo que evitar eso un poco. (57 años, mujer, taller 2) 
Nos apartan me parece, nos hacen a un lado. A la mayoría de la gente no le gusta lidiar con eso. No les gusta estar cerca del sufrimiento. A mí mismo no me gusta, pero convivo con mi patología. (35 años, varón, taller 4)

Por otro lado, se preguntó a los entrevistados qué creían que podían hacer ellos por una persona con enfermedad mental y qué creían que podía hacer una persona con enfermedad mental por ellos. Respecto a la primera pregunta, la mayoría de las respuestas estuvieron relacionadas con acompañar, ayudar, escuchar:

Ayudarla, sacarla a tomar sol, a respirar, mostrarle que hay otras cosas además del sufrimiento que vivieron. (42 años, mujer, taller 8)

Hacerla sentir bien, una caricia, afecto, una ocupación. (38 años, mujer, taller 2)

Comprenderla, orientarla. (69 años, mujer, taller 1)

Solo una minoría de los entrevistados señaló que había que tratarlas como a cualquier otra persona:

Tratarlas de igual a igual. (35 años, mujer, taller 4)

Tratarlo como exactamente igual a mí. (32 años, varón, taller 6)

Respecto a lo que los entrevistados creían que una persona con enfermedad mental" podía hacer por ellos, es de señalar que la pregunta causó sorpresa en la mayoría de los entrevistados. La mitad se refirió a respuestas categorizadas como ayudar, acompañar, escuchar:

Acompañarme, con la compañía me conformo. (57 años, mujer, taller 2)

Ser amigo, mostrarme la vida con otros ojos (más oscura y triste). (20 años, varón, taller 6)
Compartir cosas. (54 años, mujer, taller 4)

Escucharme y ayudarnos uno al otro, darme contención. (27 años, varón, taller 5)

Otras respuestas se refirieron a brindar afecto y cariño:

...te brinda cariño. (47 años, varón, taller 6)

...la parte afectiva. (69 años, mujer, taller 1)

...recibís cariño. (42 años, mujer, taller 8)

Por otro lado, se compararon las respuestas de cada entrevistado a estas dos últimas preguntas, encontrándose en cerca de la mitad una correspondencia entre lo que creían que ellos podían hacer por una persona con "enfermedad mental" y lo que esta persona podía hacer por ellos. Por ejemplo, un entrevistado respondió a la primera pregunta: "de lo poco que he aprendido acá es escucharlas, tienen necesidad de conversar y compartir" y a la segunda "esto que aprendí es mutuo"; otro entrevistado señaló frente a la primera pregunta "hacerla sentir bien, una caricia, afecto, una ocupación" y frente a la segunda "lo mismo".

\section{DISCUSIÓN}

Algunos datos sociodemográficos de las personas entrevistadas podrían dar cuenta de que una de las razones por las cuales la población utiliza servicios como el centro comunitario es la falta de apoyo y, en consecuencia, la búsqueda de espacios de vinculación social. Los datos en los cuales se fundamenta esta hipótesis son que el $80 \%$ de estas personas eran solteras, viudas o divorciadas y que, aunque un $80 \%$ vivía con su familia, solo un $7 \%$ lo hacía con su familia nuclear. También, que solo un 33\% eran trabajadoras en ejercicio. Es decir, es posible observar algunos indicios de que dos de las principales fuentes de vinculación social, el trabajo y la familia, podrían estar disminuidos entre quienes asisten al centro. Como se ha señalado en diversos estudios ${ }^{(20,21,22,23)}$ 
el apoyo social es una fuente fundamental de bienestar para las personas, y su ausencia se correlaciona con diversas causas de morbilidad y mortalidad. Por ejemplo, la carencia de una pareja se correlaciona con estrés, depresión y baja satisfacción con la vida ${ }^{(24)}$, y el desempleo y el empleo informal se correlacionan con ansiedad y depresión ${ }^{(25,26)}$. A su vez, se ha señalado que los efectos del apoyo dependen parcialmente de quien provea ese apoyo $^{(27)}$. Las características familiares y laborales de las personas entrevistados podrían dar cuenta de una reducción del apoyo provisto por pares (por ejemplo, en términos de rol) y ello podría ser uno de los aspectos que se producirían a partir de la participación en el centro, en el sentido de entrar a formar parte de un grupo de pares.

A su vez, y a pesar de tratarse de una muestra pequeña, resulta interesante hacer una lectura de género de los datos: los varones que asisten al centro están menos vinculados al trabajo que las mujeres, pese a que el porcentaje es bajo en ambos casos; y las pocas personas que vivían con su familia nuclear eran en su totalidad mujeres. Aunque la mayoría de las personas entrevistadas, así como de las personas que asisten al centro comunitario, eran mujeres, cabe señalar que, comparativamente, pareciera que los varones que acuden al centro se encuentran mucho más fragilizados que las asistentes mujeres. Como se ha planteado desde los estudios de género, los fracasos laborales afectan más gravemente la autoestima de los varones que la de las mujeres, en tanto ponen en duda su identidad de género ${ }^{(28)}$. En este sentido, cabe también una reflexión respecto de los programas y servicios, dado que muchas veces estos presentan una oferta mucho más accesible en términos materiales y simbólicos para las mujeres, lo cual constituye una barrera de acceso para los varones ${ }^{(29)}$.

Por otro lado, alrededor de un $25 \%$ de las personas entrevistadas dijeron que el motivo para empezar a asistir al centro fue la búsqueda de alguna ayuda frente a malestares ligados a la soledad y al aislamiento, considerados teóricamente como sufrimientos y malestares de época ${ }^{(30)}$, que no pueden ser reducidos o catalogados como una "enfermedad"(31). Otra buena parte de los entrevistados explicó que habían Ilegado al centro a partir de la sugerencia de profesionales, lo cual permite hipotetizar que estos últimos están contemplando para sus derivaciones servicios como este, lo que indicaría que se han empezado a incluir dentro del espectro de tratamiento actividades no ligadas de manera clara a lo tradicionalmente terapéutico. Lo anterior podría pensarse como una posible modificación en las prácticas, que coincide con lo que se ha planteado a nivel discursivo en documentos internacionales desde hace varias décadas respecto a las modalidades de atención en salud mental ${ }^{(32)} y$, recientemente, en Argentina, a partir de la ya mencionada Ley Nacional de Salud Mental ${ }^{(15)}$.

Pese a que el motivo inicial para asistir al centro en la mayoría de los casos no estuvo ligado al establecimiento de vínculos, sí es lo que motiva a las personas para continuar asistiendo, y lo que resaltaron como uno de los aspectos para los cuales les ha servido concurrir a este espacio. En este sentido, podría señalarse que un servicio como el centro comunitario, al fomentar el establecimiento de lazos sociales, ayuda a que las personas salgan del encierro y la soledad; problema que, con sus diferencias, comparten tanto las personas tradicionalmente tratadas por las instituciones psiquiátricas como aquellas que, sin haberlo estado y sin requerir de un "tratamiento" psicológico o psiquiátrico, también lo padecen. Se trataría en ambos casos de personas que han sufrido de distintos tipos de encierro, y que necesitan, como un aspecto para su recuperación o bienestar, salir de esa situación mediante la conformación de redes sociales. Es de destacar, como señala Grandesso, que al contar con el soporte y el apoyo de un grupo, las personas tienden a padecer menos frente a distintas situaciones de la vida cotidiana ${ }^{(33)}$. Además, estudios realizados específicamente con personas con trastorno mental severo muestran que la participación en espacios con otros les permite potenciar las capacidades que tienen y alcanzar una vida más autónoma ${ }^{(34)}$. 
A su vez, el hecho de que muchos de los asistentes al centro mantengan los vínculos que establecen allí, por fuera de este, da cuenta de que un componente importante de su intervención radica en promover la producción de relaciones que posibilitan el apoyo informal entre las personas, aspecto de central importancia dado que una de las características de tal tipo de apoyo, a diferencia del apoyo formal, es la reciprocidad ${ }^{(35)}$. Al respecto se ha señalado que, para las personas y su bienestar, es tan importante recibir apoyo, como darlo ${ }^{(22)}$ y que, en el caso específico de las personas con trastorno mental severo, una capacidad que les es escasamente reconocida es la de poder ayudar a otros ${ }^{(36)}$. En este sentido, los resultados indicarían que un aspecto que se observa en personas que se relacionan con sujetos con trastorno mental severo, en el contexto del centro, es la noción de reciprocidad en los vínculos, y la idea de que tales personas pueden ser tanto objeto de apoyo como fuente del mismo, en particular, de funciones ligadas a la compañía social y el apoyo emocional.

Que los entrevistados consideren a las personas con "enfermedad mental" como sujetos de los que también pueden recibir cosas puede ser visto como un avance en la desarticulación del estigma de la peligrosidad que pesó históricamente sobre el entendimiento de la "enfermedad mental". No obstante, cabe señalar que, entre aquellos que hablaban de la "enfermedad mental" en primera persona, no fue tan frecuentemente reconocida esta capacidad de ayudar a otros, lo cual podría dar cuenta de los efectos del estigma en la subjetividad de quienes la padecen.

Por otra parte, es de resaltar que gran parte de los asistentes al centro (el $80 \%$ ) conocía al menos a una persona que estuvo internada en un hospital psiquiátrico y que más de la mitad la conoció en el centro comunitario, lo cual indica que este es un medio importante para entrar en interacción con personas con trastorno mental severo. Lo anterior aporta a la discusión respecto de los efectos de la organización de los programas y servicios de salud mental, en el sentido de analizar lo que produce su exclusividad o su diversidad o, dicho en otros términos, su integración a otros servicios, o su diferenciación y su especialización ${ }^{(37)}$.También los resultados sugieren que, a partir de la interacción con personas con "enfermedad mental", se produce un cambio positivo en las percepciones de los asistentes acerca de estas. En esa línea, existen investigaciones que muestran que el contacto directo con personas con "enfermedad mental" es una de las mejores formas de neutralizar la presencia del estigma y la discriminación de la sociedad hacia ellas ${ }^{(38)}$. Sin embargo, cabe considerar que, al tratarse de un programa de externación de un hospital psiquiátrico de mujeres, la interacción se produce principal, aunque no exclusivamente, con mujeres exinternadas, en su mayoría de más de 50 años de edad, aspectos que pueden estar relacionados con la disminución en la idea de peligrosidad, la cual suele estar más asociada a varones jóvenes.

Es necesario destacar también que, pese a que las respuestas de los entrevistados dan cuenta de una reducción de prejuicios y el desarrollo de aprendizajes a partir de la interacción con personas con "enfermedad mental", los entrevistados parecieran estar más del lado de la integración que de la inclusión social. Aunque la inclusión social sea el horizonte de trabajo hacia el cual se dirigen las acciones del centro comunitario, lograr esa inclusión desborda sus posibilidades de intervención pues, al ser la exclusión social un proceso multidimensional, se requiere de diversas acciones e intervenciones, planeadas y espontáneas, para alcanzar la inclusión social. En este sentido, podría señalarse que, gracias a los vínculos que fomenta, el centro comunitario contribuye a la movilización de algunas personas desde una zona de exclusión hacia una zona de vulnerabilidad (39), ya que pese a seguir presentes determinadas fragilidades y precarizaciones, se ha logrado romper con el aislamiento social y, en este sentido, incidir en un componente necesario, mas no suficiente, para lograr la inclusión social: la reciprocidad en los vínculos ${ }^{(40)}$.

Finalmente, cabe señalar que la investigación presentada no constituye en sentido 
estricto un estudio relacional, en la medida en que solo indagó la perspectiva sobre las relaciones de uno de los actores del escenario en cuestión: la comunidad. Como se hiciera mención anteriormente, esta constituye un actor sobre el cual no suele investigarse, paradójicamente, en programas y servicios de salud mental comunitaria o de inclusión social, los cuales, al considerar de cierto modo dicha perspectiva, exploran las visiones de familiares y profesionales de los usuarios o pacientes ${ }^{(41)}$ posiblemente por las dificultades metodológicas de operacionalizar lo que es "la comunidad". Ligado a lo anterior, y si bien el enfoque relacional constituye un avance respecto de centrarse exclusivamente en las personas con "trastorno mental", podría plantearse que una paradoja presente en el modelo es la de mantener una lógica dicotómica, por ejemplo, entre la comunidad y tales personas. No obstante lo anterior, el desarrollo de una perspectiva relacional de las intervenciones en salud mental permitiría comprender cómo estas tienen beneficios tanto para las personas que tradicionalmente son consideradas como su población objetivo, como para las comunidades en las cuales transcurre su vida cotidiana. Posiblemente, este cambio de perspectiva permitiría a los trabajadores del campo considerar que de lo que se trata su quehacer es de desarrollar grupos y comunidades humanas más diversas, y no solamente de "integrar" a los diferentes.

\section{AGRADECIMIENTOS}

Los autores agradecen al Programa de Rehabilitación y Externación Asistida del Hospital Interzonal José A. Estéves por su ayuda para la realización del trabajo de campo y a la licenciada Valeria Canales por su participación. Este trabajo fue financiado por la Universidad Nacional de Lanús, mediante el proyecto de investigación No. 33A103, titulado "Evaluando las transformaciones producidas por la externación de pacientes psiquiátricos en las comunidades en las cuales viven: Percepciones desde la comunidad".

\section{REFERENCIAS BIBLIOGRÁFICAS}

1. Goldberg RW, Rollins AL, Lehman AF. Social network correlates among people with psychiatric disabilities. Psychiatric Rehabilitation Journal. 2003;26(4):393-402.
2. Saraceno B. La liberación de los pacientes psiquiátricos: De la rehabilitación psicosocial a la ciudadanía posible. México DF: Pax; 1995.

3. Saraceno B, Montero F. La rehabilitación entre modelos y práctica. En: Aparicio V, (ed.). Evaluación de servicios de salud mental. Madrid: Asociación Española de Neuropsiquiatría; 1993.

4. Menéndez E. Entrevista. Revista Trabalho, Educação e Saúde. 2012;10(2):335-345.

5. Antos Arens D. What do the neighbors think now?: Community residences on Long Island, New York. Community Mental Health Journal. 1993;29(3):235-245

6. Cook JR. Neighbors' perceptions of group homes. Community Mental Health Journal. 1997;33(4):287-299.

7. Wolfensberger W, Thomas S. PASSING (Program analysis of service systems' implementation of 
normalization goals): Normalization criteria and ratings manual. 2nd ed. Toronto: National Institute on Mental Retardation; 1983.

8. De Lorenzo García R. El futuro de las personas con discapacidad: Desarrollo humano y discapacidad. Madrid: Fundación Once; 2003.

9. Organización de las Naciones Unidas. Convención sobre los Derechos de las Personas con Discapacidad. New York: ONU; 2006.

10. Palacios A. El modelo social de discapacidad: orígenes, caracterización y plasmación en la Convención Internacional de los Derechos de las Personas con Discapacidad. Madrid: Cinca; 2008.

11. Sen A. Social exclusion: concept, application and scrutiny (Social Development Papers No 1). Manila: Office of Environment and Social Development, Asian Development Bank, 2000.

12. González Uzcátegui R. Salud mental en la comunidad en América Latina: Ejemplo de programas. En: Levav I, (ed.). Temas de salud mental en la comunidad. Washington DC: Organización Panamericana de la Salud; 1992.

13. Organización Mundial de la Salud, Ministerio de Salud de Chile. Informe WHO-AIMS sobre Sistema de Salud Mental en Chile. Santiago de Chile: OMS, Ministerio de Salud de Chile; 2006.

14. Cordeiro LRO, Oliveira MS, Souza RC. Produção científica sobre os centros de atenção psicossocial. Revista da Escola de Enfermagem da USP. 2012;46(1):119-123.

15. República Argentina. Ley 26657: Ley Nacional de Salud Mental [Internet]. 2010 [citado $10 \mathrm{abr}$ 2015]. Disponible en: http://goo.gl/xDfpBg.

16. Dirección Nacional de Salud Mental y Adicciones. Plan Nacional de Salud Mental. Buenos Aires: Ministerio de Salud de la Nación; 2013.

17. Ardila-Gómez $S$, Fernández $M$, Hartfiel MI, Ares Lavalle G, Borelli M, Canales V, Stolkiner A. Convivencia con pacientes externados y sus efectos en la salud mental de la comunidad: Estudio en la provincia de Buenos Aires, Argentina, 2012-2013. Revista Facultad Nacional de Salud Pública. 2015;32(S1):73-83.

18. Ardila-Gómez S, Ares Lavalle G, Fernández M, Hartfiel MI, Borelli M, Canales V, Stolkiner A. Social perceptions about community life with people with mental illness: Study of a discharge program in Buenos Aires Province, Argentina. Community Mental Health Journal. 2015;51(1):103-110.
19. Hernández Sampieri R, Fernández C, Baptista P. Metodología de la investigación. 5ta ed. México: McGraw-Hill Interamericana; 2002.

20. Berkman LF, Glass T. Social integration, social networks, social support, and health. In: Berkman L, Kawachi I, (eds.). Social epidemiology. New York: Oxford University Press; 2000.

21. Cohen S, Gottlieb BH, Underwood LG. Social relationships and health. In: Social support measurement and intervention: A guide for health and social scientists. Oxford: Oxford University Press; 2000. p. 3-25.

22. Gracia Fuster E. El apoyo social en la intervención comunitaria. Barcelona: Paidós Ibérica; 1997.

23. House J, Landis K, Umberson D. Social relationships and health. Science. 1988;241(4865): 540-545.

24. Holt-Lunstad J, Birmingham W, Jones BQ. Is there something unique about marriage?: The relative impact of marital status, relationship quality, and network social support on ambulatory blood pressure and mental health. Annals of Behavioral Medicine. 2008;35(2):239-244.

25. Employment Conditions Knowledge Network. Employment conditions and health inequalities: Final Report to the WHO Commission on Social Determinants of Health [Internet]. 2007 [citado 10 abr 2015]. Disponible en: http://goo.gl/RSm7EE.

26. Ortiz-Hernández L, López-Moreno S, Borges G. Desigualdad socioeconómica y salud mental: revisión de la literatura latinoamericana. Cadernos de Saúde Pública. 2007;23(6):1255-1272.

27. Wan CK, Jaccard J, Ramey SL. The relationship between social support and life satisfaction as a function of family structure. Journal of Marriage and Family. 1996;58(2):502-513.

28. Burin M. Precariedad laboral, masculinidad, paternidad. En: Burin $M$, Jiménez Guzmán L, Meler I, (comp). Precariedad laboral y crisis de la masculinidad: Impacto sobre las relaciones de género. Buenos Aires: Universidad de Ciencias Empresariales y Sociales; 2007.

29. Burin M, Meler I, Birmele R. Varones: género y subjetividad masculina. Buenos Aires: Paidós; 2000.

30. Galende E. Psicoanálisis y salud mental: para una crítica de la razón psiquiátrica. Buenos Aires: Paidós; 1990. 
31. Augsburger C. De la epidemiología psiquiátrica a la epidemiología en salud mental: el sufrimiento psíquico como categoría clave. Cuadernos Médico Sociales. 2002;81:61-75.

32. Organización Panamericana de la Salud. Declaración de Caracas: Conferencia Reestructuración de la Atención Psiquiátrica en América Latina [Internet]. 1990 [citado 10 abr 2015]. Disponible en: https://goo.gl/XCZKmy.

33. Grandesso M. Terapia comunitária: uma prática pós-moderna crítica: considerações teórico-metodológicas. São Paulo: Casa do Psicólogo; 2007.

34. Kantorski LP, Coimbra VCC, Demarco DA, Eslabão AD, Nunes CK, Guedes AC. A importância das atividades de suporte terapêutico para o cuidado em um centro de atenção. Revista de Enfermagem e Saúde. 2011;1(1):4-13.

35. Sluzki C. La red social: frontera de la práctica sistémica. Barcelona: Gedisa; 1996.

36. Albert $\mathrm{M}$, Becker $\mathrm{T}$, McCrone $\mathrm{P}$, Thornicroft G. Social networks and mental health service utilisation. A literature review. International Journal of Social Psychiatry. 1998;44(4):248-266.

37. Florenzano Urzúa R. Intervenciones de salud mental en la comunidad en programas generales de salud. En: Levav I, (ed). Temas de salud mental en la comunidad. Washington DC: Organización Panamericana de la Salud; 1992.

38. Chuaqui J. Percepción subjetiva de vecinos y tutores de personas con esquizofrenia. Psiquiatría y Salud Mental. 2004;21(2-3):130-138.

39. Jiménez Ramírez M. Aproximación teórica de la exclusión social: Complejidad e imprecisión del término: Consecuencias para el ámbito educativo. Estudios Pedagógicos. 2008;34(1):173-186.

40. Subirats J, (dir). Pobreza y exclusión social: Un análisis de la realidad española y europea. Barcelona: Fundación "La Caixa"; 2004.

41. Wald G. Promoción de la salud a través del arte: estudio de caso de un taller de fotografía en "Ciudad Oculta", la villa No 15 de la Ciudad de Buenos Aires. Salud Colectiva. 2009;5(3):345-362.

\section{NOTAS FINALES}

[a] Existe un debate en el campo de la salud mental sobre los conceptos de trastorno mental severo, enfermedad mental, sufrimiento psíquico y padecimiento mental. Excede a este trabajo entrar en dicho debate, por lo que se optó aquí por el uso del término "trastorno mental severo" puesto que goza de cierto consenso a nivel internacional. 